\title{
The timing and extent of intraosseous hypoxia in the oxidative stress-induced rat osteonecrosis model ${ }^{*}$
}

\author{
Toru Ichiseki ${ }^{1 \#}$, Ayumi Kaneuji ${ }^{1}$, Seiji Kaneko ${ }^{1}$, Syusuke Ueda $^{1}$, Yoshimichi Ueda ${ }^{2}$, Hideto Yonekura ${ }^{3}$, \\ Kiyokazu Fukui ${ }^{1}$, Tadami Matsumoto ${ }^{1}$ \\ ${ }^{1}$ Department of Orthopaedic Surgery, Kanazawa Medical University, Uchinada, Ishikawa, Japan \\ ${ }^{2}$ Department of Pathophysiological and Experimental Pathology, Kanazawa Medical University, Uchinada, Ishikawa, Japan \\ ${ }^{3}$ Department of Biochemistry, Kanazawa Medical University, Uchinada, Ishikawa, Japan \\ Email: ${ }^{*}$ tsy-ichi@kanazawa-med.ac.jp
}

Received 6 June 2013; revised 6 July 2013; accepted 18 July 2013

Copyright (C) 2013 Toru Ichiseki et al. This is an open access article distributed under the Creative Commons Attribution License, which permits unrestricted use, distribution, and reproduction in any medium, provided the original work is properly cited.

\begin{abstract}
Using a rat oxidative stress-induced femoral head osteonecrosis model, we determined the presence/ absence and timing of the generation of hypoxia in the femoral head. DL-Buthionine-(S,R)-sulfoximine (BSO) $500 \mathrm{mg} / \mathrm{kg}$ was administered intraperitoneally to male Wistar rats. The rats were killed at $1,3,6,12$ hours, and 1, 3, 5 days after BSO administration, and the bilateral femora were removed. A group not administered BSO (control group) was also studied (each group $n=5$ ). In the femoral heads of each group, the expression of hypoxia-inducible factor-1 alpha (HIF-1 $\alpha$ ) as an index of hypoxia was confirmed by the Western blot method, and quantified using analytical software. In the femoral head increased HIF-1 $\alpha$ expression was found in all groups from 1 hour after BSO administration $(p<0.05)$. In particular, in all specimens of the group 3 hours after BSO administration the most intense expression of HIF-1 $\alpha$ amounting to about 13-fold of that of control group was noted $(p<0.001)$. The present results suggested that in the extremely short period of 3 hours after BSO administration hypoxia severe enough to cause osteonecrosis was induced by oxidative stress in the rat femoral head.
\end{abstract}

Keywords: Osteonecrosis; Oxidative Stress; Buthionine Sulfoximine; Hypoxia-Inducible Factor- $1 \alpha$

\section{INTRODUCTION}

A broad consensus has been reached implicating intraos-

${ }^{*}$ Conflict of interest: No conflict of interest has been declared by the authors.

${ }^{\#}$ Corresponding author. seous ischemia in the development of steroid-induced femoral head osteonecrosis, with this likely caused by a single ischemic event. However, few investigations so far have focused on issues such as the amount and timing of the ischemia that occurs. To better clarify the pathogenesis of this condition, further studies are required to characterize the development of hypoxia in bone.

Recently, in vivo oxidative stress has been implicated in the development of steroid-induced osteonecrosis [1-3]. In addition, the efficacy of antioxidant in the prevention of steroid induced osteonecrosis has been reported in animal models [4-6]. Our group previously reported a model in which osteonecrosis is induced in the rat femoral head by oxidative stress after a single intraperitoneal injection of DL-Buthionine-(S,R)-sulfoximine (BSO), an inhibitor of glutathione (GSH). In this model, femoral head osteonecrosis develops 7 days after BSO administration in $40 \%$ of rats [7].

Since in the rat, histological study shows that osteocytes develop empty lacunae about $96 \mathrm{~h}$ after the onset of ischemia $[8,9]$, it is thought that hypoxic events leading to osteonecrosis in an oxidative stress-induced rat osteonecrosis model occur in the bone at least by $3-5$ days after the ischemia. With this in mind, using as an index the transcription factor, hypoxia-inducible factor-1 alpha (HIF-1 $\alpha$ ), which has been widely studied as an index of hypoxia $[10,11]$, we determined the timing of the development and amount of hypoxia in the femoral head from $1 \mathrm{~h}$ to $5 \mathrm{~d}$ after the administration of BSO in a rat oxidative stress-induced femoral head osteonecrosis model [7].

\section{MATERIALS AND METHODS}

\subsection{Animals}

Forty male Wistar rats age 24 weeks (body weight 400 $450 \mathrm{~g}$ ) were studied at the Animal Center of Kanazawa 
Medical University. All rats were housed under standard laboratory conditions (temperature $24^{\circ} \mathrm{C}, 12$-hour light/ dark cycle) and were given food and water ad libitum. They were given a single intraperitoneal injection of BSO (500 mg/kg) and were killed 1 hour (B1h group), 3 hours (B3h group), 6 hours (B6h group), 12 hours (B12h group), 1 day (B1d group), 3 days (B3d group) or 5 days (B5d group) after administration. Untreated rats were compared as a control group (group N). Each group contained 5 rats. This study was conducted in accordance with the guidelines of the Animal Research Committee of Kanazawa Medical University.

\subsection{Tissue Preparation}

All rats were killed using intraperitoneally injected sodium pentobarbital. Promptly after sacrifice the bilateral femoral heads were extracted and cryopreserved at $-80^{\circ} \mathrm{C}$ after the bilateral femora were removed.

\subsection{Western Blot Assay}

Immunoblotting for HIF-1 $\alpha$ was performed on the femoral head of BSO administered rats, as well as non-treated rats. Proteins were extracted using lysis buffer $(50 \mathrm{mM}$ Tris- $\mathrm{HCl}, \mathrm{pH} 7.6,10 \%$ glycerol, $5 \mathrm{mM}$ magnesium acetate, $0.2 \mathrm{mM}$ ethylenediamine tetraacetic acid, $1 \mathrm{mM}$ phenylmethylsulfonyl fluoride, and 1\% sodium dodecylsulfate). Extracted protein (20 mg) was applied to and electrophoresed on a $10 \%$ polyacrylamide gel, and transferred to a nitrocellulose membrane (Atoh, Tokyo, Japan). The membranes were reacted overnight at $4{ }^{\circ} \mathrm{C}$ with anti HIF-1 $\alpha$ polyclonal antibody (Millipore) at a concentration of 1:200 dilution. After incubation with peroxidaselabeled goat anti-rabbit IgG antibody (Dako Cytomation) for 1 hour at room temperature and vigorous washing, the nitrocellulose membrane was incubated with ECLpulse (GE Medical, USA) and photographed digitally using LAS4000 (FUJIFILM, Tokyo, Japan). All samples were standardized by immunoblot using anti- $\beta$ actin mouse monoclonal antibody (Sigma Chemical Co., St. Louis, MO).

\subsection{Quantification of the Western Blot Results}

Protein quantification was done using analytical software "Multi Gauge" (FUJIFILM, Tokyo, Japan). $\beta$-actin was used as the internal control for all specimens in each group. All values were expressed as the mean $\pm \mathrm{SD}$, and Arbitrary Units (AU) were used at the time of measurement.

\subsection{Statistical Analysis}

Statistical analysis was performed using analysis of variance with Dunnett's multiple comparison test. By using this test we were able to determine the point in time after administration of BSO that HIF-1 $\alpha$ level showed a significant change, as compared with the values in $\mathrm{N}$ group. $\mathrm{p}$ values less than 0.05 were considered significant. The statistical analysis was performed using StatView J-5.0 software (SAS Institute).

\section{RESULTS}

\subsection{Western Blot for HIF-1 $\alpha$}

An approximate molecular weight of around $120 \mathrm{kDa}$ for HIF-1 $\alpha$ was demonstrated by Western blot analysis. HIF- $1 \alpha$ was overexpressed in BSO administered specimens. HIF- $1 \alpha$ expression started to increase from B1h Group, with a marked increase found in all specimens of B3h Group. From B6h-B12h Group onwards increased HIF-1 $\alpha$ expression was found as compared to N Group (Figure 1).

\subsection{Comparison by Quantification of HIF-1 $\alpha$ Expression by Western Blot}

When quantified using analytical software, the mean value was $(9.9 \pm 1.0) \times 10^{4} \mathrm{AU}$ in N Group, and $(128.6 \pm$ $12.2) \times 10^{4} \mathrm{AU}$ in $\mathrm{B} 3 \mathrm{~h}$ group, representing a significantly increased expression of about 13-fold in the latter as compared to the former $(\mathrm{p}<0.001)$. The corresponding values were $(97.0 \pm 37.0) \times 10^{4} \mathrm{AU}$ in B6h group, $(94.8 \pm 43.2) \times 10^{4} \mathrm{AU}$ in $\mathrm{B} 12 \mathrm{~h}$ group, $(74.6 \pm 31.5) \times$ $10^{4} \mathrm{AU}$ in B1d group, $(58.0 \pm 31.2) \times 10^{4} \mathrm{AU}$ in $\mathrm{B} 3 \mathrm{~d}$ group, and $(80.9 \pm 38.8) \times 10^{4} \mathrm{AU}$ in B5d group. In all of the groups administered BSO HIF expression was significantly increased as compared to $\mathrm{N}$ group $(\mathrm{p}<0.05)$ (Figure 2).

\section{DISCUSSION}

Various theories have been proposed to explain the pathogenesis of steroid-induced osteonecrosis [12-14]. As noted above, while there is general agreement that in the end osteonecrosis is induced by intraosseous ischemia, various issues such as the timing of occurrence and amount of the intraosseous hypoxia remain unclear. To elucidate the developmental mechanisms of osteonecrosis

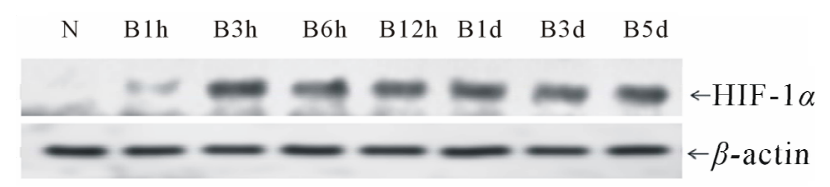

Figure 1. HIF-1 $\alpha$ expression by Western blot. In N group almost no HIF- $1 \alpha$ expression was found. HIF- $1 \alpha$ expression started to increase from B1h group, with a marked increase found in all specimens of B3h group. From B6h group onwards increased HIF-1 $\alpha$ expression was found as compared to $\mathrm{N}$ group. In all specimens $\beta$-actin was used as an internal control. 


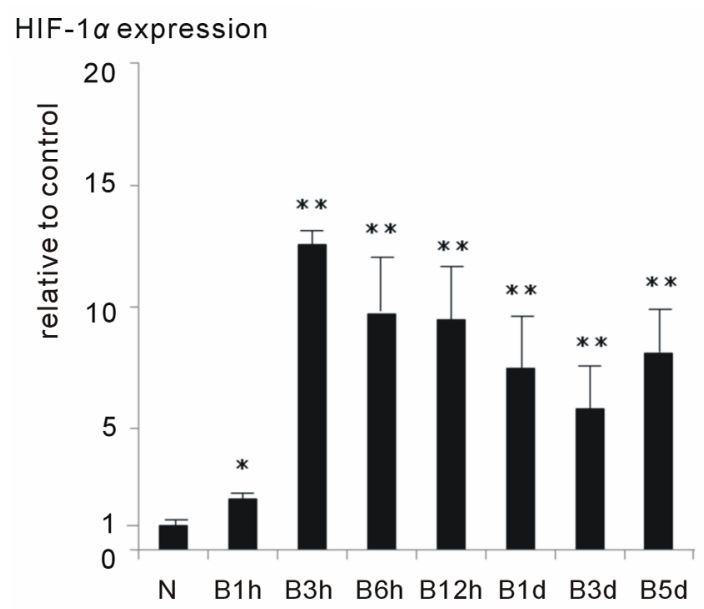

Figure 2. Quantification of results by Western blot. In the comparison with $\mathrm{N}$ group, increased HIF1 $\alpha$ expression was found in all the groups administered BSO. HIF- $1 \alpha$ expression in B3h group was about 13fold of that of $\mathrm{N}$ group, with the most intense hypoxia occurring at this time $\left({ }^{*} \mathrm{p}<0.05\right.$ vs $\mathrm{N}$ group, ${ }^{* *} \mathrm{p}<$ 0.001 vs $\mathrm{N}$ group).

and devise optimal prophylactic strategies it is important to grasp the changes occurring in bone from immediately after drug administration. For this reason, detailed determination of the actual severity of the ischemic event occurring after drug administration and pinpointing the stage at which it occurs, as well as confirmation of the previously described finding that only a single ischemic event is induced are required.

In the present study, our attention was drawn to the transcription factor HIF- $1 \alpha$ as an index of tissue hypoxia. HIF- $1 \alpha$ is particularly well suited to assess the timing and amount of hypoxia because while it is rapidly degraded in the presence of normal oxygen concentrations, in the presence of hypoxia it is not degraded and its concentration rises [10,11,15-17].

In the rat, osteocyte apoptosis has been reported to begin $12 \mathrm{~h}$ from the ischemic insult, with osteonecrosis observed 4 - 5 days from the onset of ischemia $[8,9]$. Accordingly, in this investigation we considered it important to focus on the state of the hypoxia occurring in the bone up to 5 days after BSO administration at which point the findings of osteonecrosis can be confirmed histopathologically in this model [7].

This experiment confirmed that in the model used hypoxia in the femoral head was induced by oxidative stress within the extremely short period of $<3 \mathrm{~h}$. Also, especially in B3h group as compared to $\mathrm{N}$ group about a 13 -fold increase in HIF-1 $\alpha$ expression was found. The expression of HIF- $1 \alpha$ has been reported to increase by about $\geq 10$-fold in hypoxia of about $2.5 \%-5 \%$ of the normal oxygen concentration [15-17]. Accordingly, we concluded that in this model after BSO administration the oxygen concentration of femoral head tissues in all specimens fell to less than about $2.5 \%-5 \%$ of normal within $3 \mathrm{~h}$.

The results of this study are extremely important in considering the developmental mechanism of femoral head osteonecrosis. This is because if a hypoxic event is indeed the ultimate cause of osteonecrosis, all specimens in this model would be expected to develop osteonecrosis. However, since the rate of development of femoral head osteonecrosis was only $40 \%$ in this model, the possible involvement of some kind of secondary factor in the development of osteonecrosis occurring after the hypoxic event $3 \mathrm{~h}$ after BSO administration is also suggested. Also, from B6h group onwards as compared to $\mathrm{N}$ group HIF-1 $\alpha$ expression was significantly enhanced. Accordingly, it could be concluded that the intraosseous hypoxic state induced by oxidative stress begins in bone from $1 \mathrm{~h}$, with an extremely strong hypoxic state developing within $3 \mathrm{~h}$, and the hypoxic state persisting from B6h group onwards albeit while showing some gradual improvement.

It has previously been reported that oxidative stress develops within $24-72$ hours after steroid administration [1-3]. The present results showing the occurrence of severe hypoxia from $3 \mathrm{~h}$ after oxidative stress help to identify the mechanism underlying the development of osteonecrosis very soon after steroid administration. Accordingly, the present experimental results can be considered extremely important also from the viewpoint of devising novel preventative approaches for steroid-induced osteonecrosis.

\section{ACKNOWLEDGEMENTS}

This work was partly supported by grant 21591963 (to TI) from the Japanese Ministry of Education, Culture, Sports, Science and Technology.

\section{REFERENCES}

[1] Ichiseki, T., Matsumoto, T., Nishino, M., Kaneuji, A. and Katsuda, S. (2004) Oxidative stress and vascular permeability in steroid-induced osteonecrosis model. Journal of Orthopaedic Science, 9, 509-519. doi:10.1007/s00776-004-0816-1

[2] Ichiseki, T., Kaneuji, A., Ueda, Y., Kaneko, S., Ueda, S. and Matsumoto, T. (2012) The initial phase of oxidative stress in a steroid-induced osteonecrosis rabbit model. Advances in Bioscience and Biotechnology, 3, 978-982. doi:10.4236/abb.2012.327120

[3] Ichiseki, T., Kaneuji, A., Katsuda, S., Ueda, Y., Sugimori, T. and Matsumoto, T. (2005) DNA oxidation injury in bone early after steroid administration is involved in the pathogenesis of steroid-induced osteonecrosis. Rheumatology (Oxford), 44, 456-460.

[4] Mikami, T., Ichiseki, T., Kaneuji, A., Ueda, Y., Sugimori, 
T., Fukui, K. and Matsumoto, T. (2010) Prevention of steroid-induced osteonecrosis by intravenous administration of vitamin E in a rabbit model. Journal of Orthopaedic Science, 15, 674-677.

doi:10.1007/s00776-010-1516-7

[5] Li, G.Y., Feng, Y., Cheng, T.S., Yin, J.M. and Zang, C.Q. (2013) Edaravone, a novel free radical scavenger, prevents steroid-induced osteonecrosis in rabbits. Rheumatology (Oxford), 52, 438-447. doi:10.1093/rheumatology/kes313

[6] Nozaki, Y., Kumagai, K., Miyata, N. and Niwa, M. (2012) Pravastatin reduced steroid-induced osteonecrosis of the femoral head in SHRP rats. Acta Orthopaedica, 83, 8792. doi:10.3109/17453674.2011.641103

[7] Ichiseki, T., Kaneuji, A., Ueda, Y., Nakagawa, S., Mikami, T., Fukui, K. and Matsumoto, T. (2011) Osteonecrosis development in a novel rat model characterized by a single application of oxidative stress. Arthritis \& Rheumatism, 63, 2138-2141. doi:10.1002/art.30365

[8] Sato, M., Sugano, N., Ohzono, K., Nomura, S., Kitamura, Y., Tsukamoto, Y. and Ogawa, S. (2001) Apoptosis and expression of stress protein (ORP150, HO1) during development of ischaemic osteonecrosis in the rat. The Journal of Bone \& Joint Surgery, 83-B, 751-759. doi:10.1302/0301-620X.83B5.10801

[9] Mary, C. (1965) A histological study of avascular necrosis of the femoral fracture. The Journal of Bone \& Joint Surgery, 47-B, 749-776.

[10] Hirota, K. and Semenza, G.L. (2005) Regulation of hy- poxia inducible factor 1 by prolyl and asparaginyl hydroxylases. Biochemical and Biophysical Research Communications, 338, 610-616. doi:10.1016/j.bbrc.2005.08.193

[11] Semenza, G.L. (2001) HIF-1, O2, and the 3 PHDs: How animal cells signal hypoxia to the nucleus. Cell, 107, 1-3. doi:10.1016/S0092-8674(01)00518-9

[12] Jones Jr., J.P. (1993) Fat embolism, intravascular coagulation, and osteonecrosis. Clinical Orthopaedics and Related Research, 292, 294-308.

[13] Jones Jr., J.P. (1994) Concepts of etiology and early pathogenesis of osteonecrosis. Instructional Course Lectures, 43, 499-512.

[14] Wang, G.J., Sweet, D.E., Reger, S.I. and Thompson, R.C. (1977) Fat-cell changes as a mechanism of avascular necrosis of the femoral head in cortisone treated rabbits. The Journal of Bone \& Joint Surgery, 59, 729-735.

[15] Vaupel, P. (2004) The role of hypoxia-inducible factors in tumor progression. Oncologist, 5, 10-17. doi:10.1634/theoncologist.9-90005-10

[16] Rankin, E.R. and Giaccia, A.J. (2008) The role of hypoxia-inducible factors in tumorigenesis. Cell Death Differ, 15, 678-685. doi:10.1038/cdd.2008.21

[17] Jiang, B.H., Semenza, G.L., Bauer, C. and Marti, H.H. (1996) Hypoxia-inducible factor 1 levels vary exponentially over a physiologically relevant range of $\mathrm{O} 2$ tension. American Journal of Physiology, 271, 1172-1180. 